\title{
Research Staff Postal Address
}

National Cancer Institute

\section{Source}

National Cancer Institute. Research Staff Postal Address. NCI Thesaurus. Code C94065.

A contact point used to send physical forms of communication to the research staff. 